\title{
Gini in A Bottle: A Case Study of Pareto's Principle in the Wild
}

\author{
Alex Blate ${ }^{1}$, Kevin Jeffay ${ }^{2}$ \\ ${ }^{1}$ Doctoral Student, Department of Computer Science, University of North Carolina at Chapel Hill \\ ${ }^{2}$ Gillian T. Cell Distinguished Professor, Department of Computer Science, University of North Carolina at \\ Chapel Hill \\ E-mail: 'blate@cs.unc.edu, ${ }^{2}$ jeffay@cs.unc.edu
}

\begin{abstract}
This paper presents a case study of socio-economic disparities - human factors - having tremendous impact on the performance and behavior of a cloud-based software system. Failures to take such factors into account lead to serious design, implementation, and operational problems. A detailed empirical analysis of a commercial mobile network address book web application, serving over 8.3 million subscribers, was conducted for the joint purposes of building realistic subscriber behavior and data models and to explain certain performance characteristics and expectations. Extensive analysis of anonymized production data revealed that many aspects of users' data and activity exhibited strongly-heavy-tailed characteristics, particularly characteristics affecting database performance and interactive request latencies, which could be ameliorated by traditional techniques such as caching or multi-threading. Several performance-critical aspects of users' data were found to be well-described by the Log-Normal probability distribution, were heavily-skewed to the right, and exhibited Gini coefficients consistent with income inequalities in the Western world. The analytical model was translated into enhanced simulation and performance tooling, enabling more realistic performance and capacity testing of the product. Our deeper understanding also lead to changes in monitoring and system performance evaluation and quality-of-service parameters, statements of performance and capacity ratings, and architecture that would not otherwise have been justifiable.
\end{abstract}

Keywords: Human Factors, Long Tail, Scalability, Carrier Applications, Performance

\section{INTRODUCTION}

The design, modeling, operation, and analysis of software systems must take into account the often long- or heavy-tailed effects due to human endusers. In many cases, the quantity, frequency, and nature of use of a given system will vary greatly between different users and, as we will show, these effects can magnify each other. For example, distributions of file sizes have long been known to be heavy-tailed and, in some studies, it has been shown that the majority of disk space is consumed by a small percentage of files [1]. Further analysis of the nature of human-generated files - how they grow and change over time - shows that, analytically, file sizes should be expected to follow a classically heavy-tailed distribution (Log-Normal, in this case) [2]. Since the early days of the public Internet, it has been shown that a number of important aspects of web traffic - including HTTP response sizes, inter-request times, etc. - also exhibit self-similar behavior [3].

The present case-study examines the collision between a large software system and a complex of changes in end-user behavior. The system in question is a cloud-based service provided by a mobile carrier for the backup and synchronization of and web access to the address books on users' mobile devices. The service is used, for example, to migrate a user's address book to a newly-purchased device, restore the address book in the case of a lost or damaged device, or synchronize contents between multiple devices.

For many years of the deployment, per-user storage and computing requirements (required for managing performance and capacity) were wellmodeled with simple uniform distributions about some empirically-derived mean (average). In essence, the per-user resource demand could be considered a constant for the purposes of performa- 
nce, capacity, and SLA (Service Level Agreement) computations. Progressive changes in user behavior, due to, among other things, the availability of more powerful and integrated endpoints and the addition to the system of realtime user-interactive features, resulted in pathologically non-uniform user demand and the failure of simple per-user resource demand models to predict real-world behavior.

"Problems" began to arise in the production system. For example, the key performance indicators (KPIs) used by system administrators included the maximum and average duration of a device synchronization transaction (wherein, the device and the service exchange and synchronize content). Over several months, maximum transaction times skyrocketed and averages began exceeding defined limits. Acting under the assumption of a uniform user base, these issues were attributed to software or architectural problems (e.g., some change had made an operation less efficient or some component was failing to scale as advertised, causing congestion). An incorrect model of user demand (the uniform model) lead to the identification of "problems" that really were correct and expected behavior (given a correct understanding of the user base).

The actual problem was that the users' behavior had diverged substantially from anyone's expectations: the changes in performance metrics were a symptom. This is exemplified below ( $§ I V)$ via a careful analysis of access logs from the web services back-end of the user portal - which analysis showed that response times were stronglycorrelated with response sizes (and, therefore, the amount of work done by the system) and that the distribution of response times and sizes themselves were heavy-tailed. Several similar analyses of other metrics lead to the same conclusions.

Our burden, then, was to prove our hypothesis: that the heavy-tailed distributions of performance metrics were driven by underlying human factors: properties of the users, their behavior, and their data. Subsequent analysis of anonymized database dumps showed a very wide disparity in per-user resource demand. We also found that "expensive" users (mainly those with large numbers of contacts) used the system more often and were more likely to visit the web portal - directly-exposing these users

We place the word "problems" in quotations because the observed phenomena were really symptoms - a reflection of changing human interaction with the system. The problem proper was lack of planning and adaptation to the changing demands on the system. to high-latency transactions.

With a clear understanding of the model - now known to be closely-approximated by the LogNormal distribution, the interpretation of the "problems" (symptoms) took the form of a need to mitigate or optimize around the now-expected long processing times experienced by a small but vocal subset of the user base.

\section{APPLICATION AND USER POPULATION}

The software system discussed herein is a SaaS (Software as a Service) [4] application deployed in a major mobile phone carrier. The service itself provides backup and synchronization of the address books on mobile phones as well as a web portal where users can access and manipulate their address books. The service was available to all of the carrier's users, supported on all of the carrier's handsets (from low-end "free phones" to expensive smart phones and PDAs) and, thus, reached a wide cross-section of the population. At the time of the present analysis, the system served over 8.3 million subscribers with over 1 billion contacts (address book entries).

A very high-level block diagram of the service is shown in Figure 1. The details of the operation of the system are not salient for the present discussion; note, however, that transactions through the system may touch multiple databases and that synchronization operations, in particular, read and write files from an NFS volume. Said more succinctly, most operations in the system are heavily I/O-bound.

Each user's "cost" in the system - including processing time and storage - is directlyproportional to the size of the user's address book, how many devices he has, and how many and how frequently changes are made to his address book. Finally, modulo resource contention, users and their transactions are independent (i.e., one user's transactions and data do not affect those of other users).

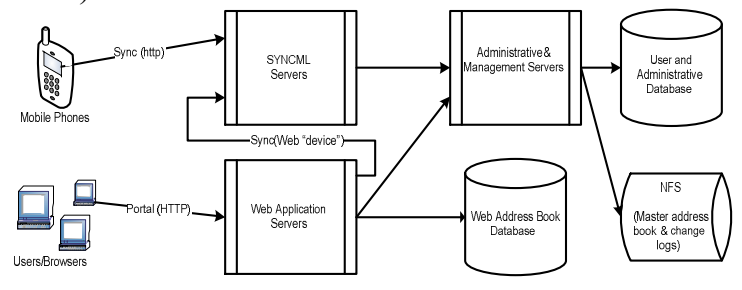

Fig. 1. Block diagram of SaaS address book service. transaction, lower-bounds transaction time. This is a structural property of the system in question.

Because of inter-user transaction data 
independence and users' relatively infrequent use of the system (a few times per day, at most), caching - at almost any point in the system - could not improve performance. Synchronization operations require the sequential processing of contact records and changes; parallelization was deemed unlikely to be effective. Thus, many of the commonly-used techniques to mitigate transaction latency were of no use to us.

This service was first deployed at a time when mobile devices had relatively limited storage capacity (perhaps 64-256 phone numbers) and did not, for example, integrate with corporate address books (e.g., Microsoft Exchange ${ }^{\mathrm{TM}}$ ), social media (e.g., LinkedIn ${ }^{\mathrm{TM}}$ ), and the like that automatically add contacts to one's address book. Effectively, there was a hidden upper bound on address book size (for most users) because it was more timeconsuming to add contacts via handset keypads. ${ }^{2}$ Mobile minutes were also more expensive at this time, possibly accounting lower utilization and adoption.

The subscriber population was modeled (fairly effectively) by assuming a constant or uniformlydistributed per-user address book size (e.g., address book sizes are uniformly-distributed over [0,200]). Essentially, all users were presumed to be equal. This model was used for performance and capacity planning and testing of computing and storage resources in Figure 1, generating SLA values, and the model was presumed when interpreting monitoring data and KPIs. More specifically, system performance was characterized by common first-order descriptive statistics such as minimum, maximum, and average. The system was deemed to be experiencing "problems" if, for example, average or maximum transaction times crossed some high-water mark. This model and methodology served the carrier well for a number of years.

\section{DISPARITY APPEARS}

In retrospect, we identified two changes in user behavior - one caused by a product change and one caused by technological evolution - that necessitated a re-visitation and eventual revision of the subscriber model.

First, the web portal, used to view and manipulate one's address book, underwent enhancement and was made available free-of-

\footnotetext{
2 Viewed another way, we speculate that, at this time, users would have had larger address books but for these technological limitations. New features, devices, and economies opened the door to the expression of existent latent behavior.
}

charge, marketed to all subscribers (its seldom-used nascent predecessor was decommissioned), and integrated with the carrier's web portal. Up to this time, most user interactions with the system were device synchronizations, which are asynchronous and non-user-interactive. If a user replaced his handset and it took several hours for his address book to be restored, this was considered normal and was well-tolerated by users. However, as users began accessing the new web portal, long response times became highly-visible and, for some users, made the portal unusable.

Secondly, over the preceding several years, handsets had become far more sophisticated: commodity flash memory enabled much larger address books, richer content was added to the address books themselves, and newer devices could integrate with Enterprise Messaging systems (e.g., Microsoft Exchange $\left.{ }^{\mathrm{TM}}\right)$ and social media applications (such as LinkedIn ${ }^{\mathrm{TM}}$ and Facebook ${ }^{\mathrm{TM}}$ ). Higher-end devices also became more affordable and were more widely-adopted by subsets of the population such as business users and a burgeoning SMS-enamored generation. The net effect was that, at least for some users, it became both possible and easy to grow their address books well-beyond anything anticipated by the carrier or the product's architects.

We see here a complex mesh of human factors: early-adopters, power users, and others with financial means could and did upgrade to these more-capable devices; these users also tend to have higher expectations for quality-of-service (QoS). The same set of users is arguably more likely to use features such as Exchange integration or, indeed, this may have motivated their buying decision. And, possibly being more tech-savvy, these users may have been more excited to try out the shiny new Web 2.0 portal.

\section{WEB SERVICE RESPONSE TIME ANALYSIS}

User and carrier complaints about the web portal's performance led to us performing a detailed analysis of the performance of the web services upon which the user portal is built. We were provided with a large set of web access logs (akin to Apache's http_access log [11]) from production for analysis. The logs' content included request URI, response code, response time, and response size information for each request. One web service call in particular, which we will refer to as "getAllContacts" was of particular interest: it was the second-most-frequently used method, it was guaranteed to be called at least once per user login 
to the portal, and it was responsible for over $58 \%$ of response time among all web service requests. The nominal SLA for all web service calls was an average response time of less than $1,000 \mathrm{~ms}$ (milliseconds). In the given sample, the average response time for getAllContacts was $1,789 \mathrm{~ms}$ while the median was $881 \mathrm{~ms}$, the minimum was $29 \mathrm{~ms}$, and the maximum was over $548,000 \mathrm{~ms}$ (over 9 minutes).

It is worth pointing out at this point that these four descriptive statistics, alone, typically indicate a heavily-right-skewed distribution. ${ }^{3}$ Assuming that the work (and therefore time) required to serve a request is proportional to the user's address book size and assuming that address book sizes have a narrow, symmetric distribution about some mean (e.g., a uniform or normal distribution with mean 100 contacts), then we would expect the average and median response times to be approximately equal (little or no skew). A large number of "outliers" due to, say, abandoned transactions or diurnal load characteristics (i.e., load fluctuations strongly-correlated with time-of-day - higher during business hours and lower at night, for example [13]), could account for this discrepancy, as could some underlying performance problem or software defect causing requests of the same a priori "cost" to have dramatically varying response times.

To visualize the underlying phenomenon, we constructed a graph of the cumulative distribution function (CDF) of response times, depicted in Figure 2.

Recall that the average response time for getAllContacts was $1,789 \mathrm{~ms}$; this falls at about the 75 th percentile - i.e., $75 \%$ of all requests had response times less than the average. Nearly $60 \%$ of requests met the $1,000 \mathrm{~ms}$ SLA. The density of the upper tail, however, cannot be explained away as some equivalence class of "outliers".

Importantly, we also observed that, over a substantial subset of the samples, the response time divided by response size was roughly equal ${ }^{4}-$ i.e.

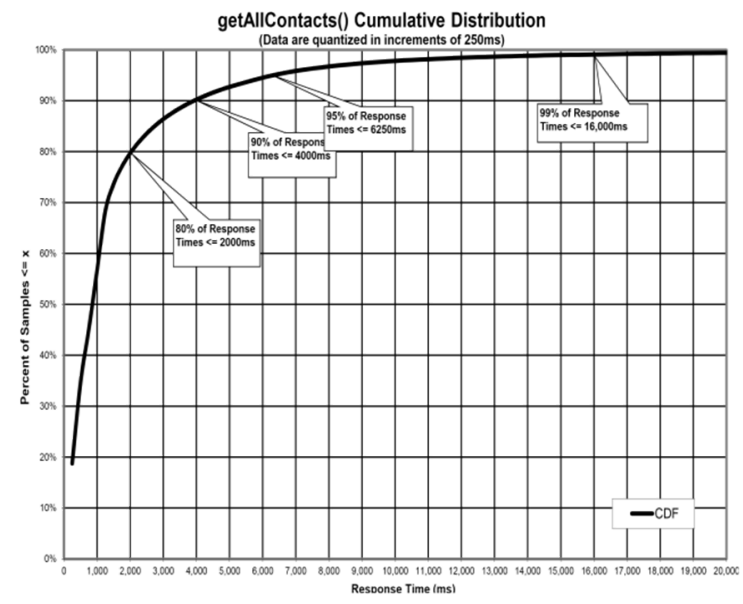

Fig. 2. Cumulative distribution of response times of getAllContacts web service call.

the response time increased linearly in proportion to the amount of data returned, which, in principle, is proportional to the amount of computation and NFS and database I/O demanded by the transaction. There was also little-to-no diurnal correlation among response times; that is, the distribution of response times did not change throughout the day response times in excess of 2,000ms (80th percentile) were seen even late at night. Our conclusion, then, was that the longer response times, however undesirable, are to be expected because more work is being done for the user.

\section{REVISITING THE MODEL}

Justifying the web service performance discussed above (and other related issues) required understanding why the workload had deviated so far from the model. For example, perhaps certain users with large address books accessed the portal more frequently, thus skewing our statistics. Or, as we asserted and subsequently proved, the user population had changed.

\footnotetext{
A simplistic and intuitive definition of skew is as a signed measure of the difference between the mean and the median. Many other, more technical definitions exist, but are outside the scope of the present discussion.

4 Intuitively, this quantity is a measure of throughput the time it takes to compute and send a given number of results. If throughput is substantially invariant, then (we infer) transaction "cost" is linearly-proportional to, in this case, the user's address book size.

5 Note: we do not argue that such response times are acceptable; rather, we conclude that the software is working as designed.
} 


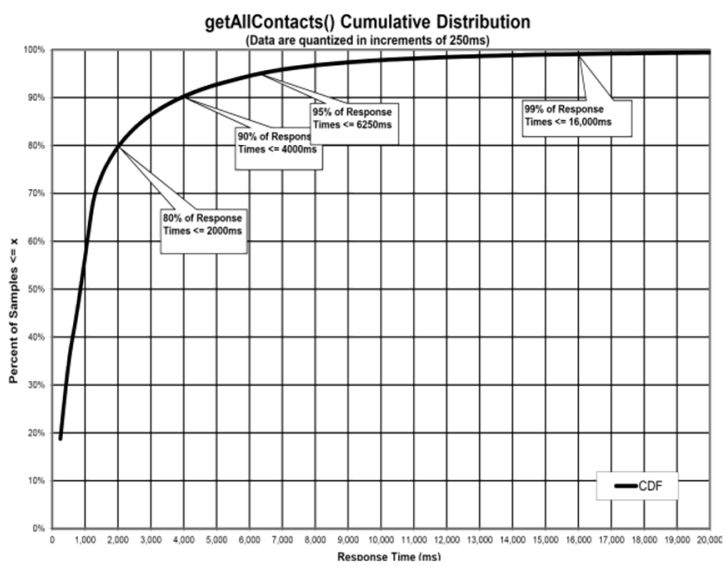

Fig. 3. Cumulative distribution of response times of getAllContacts web service call.

Table 1: Descriptive Statistics

\begin{tabular}{|l|r|}
\hline Total Subscribers & $8,329,649$ \\
\hline Total Contacts & $1,203,082,358$ \\
\hline Mean (Average) & 144.43 \\
\hline Median & 110 \\
\hline Geometric Mean & 104.80 \\
\hline Harmonic Mean & 67.24 \\
\hline Min & 1 \\
\hline Max & 32,766 \\
\hline Std. Deviation & 142.57 \\
\hline Variance & $20,325.26$ \\
\hline Log-Normal $\hat{\mu}$ & 4.6521 \\
\hline Log-Normal $\hat{\sigma}$ & 0.8427 \\
\hline
\end{tabular}

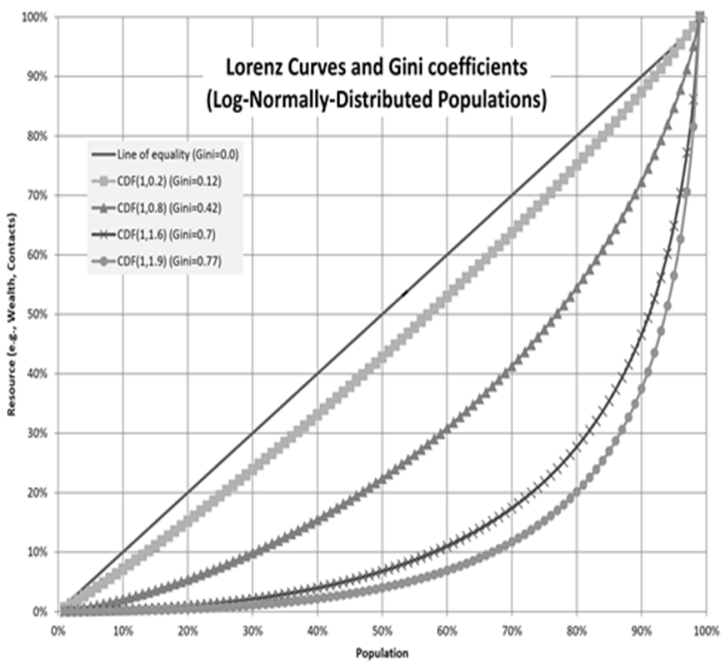

Fig. 5. Lorenz curves having differing Gini Indices. A higher Gini Index indicates a larger degree of inequality of resource distribution among the population, which is reflected by a steeper, more convez Lorenz curve.
With the carrier's permission, we were able to obtain anonymized copies of certain database tables from the production system. We proceeded to examine the distribution of address book sizes among the population, depicted in the histogram in Figure 3 and the descriptive statistics in Table 1.

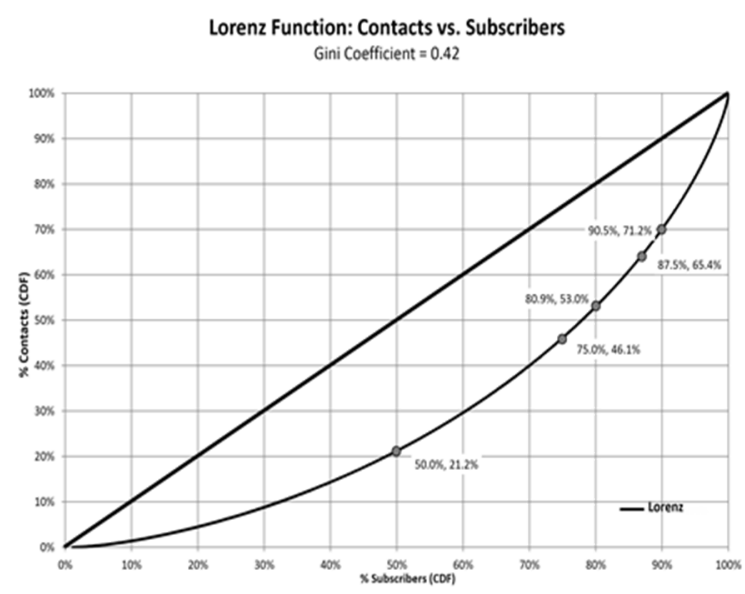

Fig. 4. Lorenz function plot of the distribution of contacts among users.

Approximately 95,000 subscribers having zero contacts were excluded from the present analysis, justified by the fact that such users are responsible for essentially zero resource consumption on the system. Due to the self-similarity of the underlying distribution, discarding these few points does not affect the subsequent analysis.

The histogram and descriptive statistics show a very right-skewed distribution (as expected) and a heavy right tail. The most common address book size (mode) is on the order of 50, the median is 110 , and the average is 144 . Note that the $\mathrm{x}$-axis would extend all the way to 32,766 (which happened to be the maximum size of the address book on a Blackberry ${ }^{\mathrm{TM}}$ device at the time). The graph was truncated for clarity.

Another way of visualizing the population is by graphing the Lorenz function [6], given by the CDF of contacts as a function of the CDF of the population, as shown in Fig. 5.

The main diagonal $(\mathrm{y}=\mathrm{x})$ is also known as the "Line of Equality" - if contacts were equally distributed among users, the Lorenz function would fall on this line. Note that, by definition, the Lorenz function can never take values above the line of equality. Lorenz curves are used in many sorts of population studies, such as the study of the distribution of income, Economics, Biology, Geology, Medicine, and the Social Sciences [5], [6]. The shape of the curve provides insight into the 
quantitative nature of inequalities.

In the case of the subscriber population, we see that the lower $50 \%$ of subscribers account for only about $21 \%$ of all contacts in the system. Moving up the curve, the lower $90 \%$ of users only account for about $71 \%$ of all contacts; conversely, $10 \%$ of the subscribers own about $30 \%$ of the contacts in the system (and thus are responsible for about 30\% of the system's load).

In population studies, the amount of inequality is sometimes expressed as the "Gini Coefficient" or "Gini Index"5 [6], which is the ratio of the area between the line of equality and the Lorenz curve and the area under the line of equality. It may also be thought of as the degree of concavity of the Lorenz curve (which is, by definition, nondecreasing and concave). A Gini coefficient of zero (0.0) indicates "perfect equality" while a Gini coefficient of one (1.0) indicates perfect inequality. ${ }^{6}$ (The latter case can be understood by considering a population where only one individual owns all the resources.)

To illustrate the interpretation of the Gini coefficient, we have constructed Lorenz curves for several Log-Normal distributions having the same scale but differing shape parameters (the LogNormal distribution is discussed in more detail below), as shown in Fig. 4.

Observe that the line of equality has a Gini index of 0.0. Intuitively, this means that resources $\mathrm{R}$ are distributed uniformly among the population, with each person owning $\mathrm{R} / \mathrm{n}$. Considering the distributions shown, we see that a higher Gini index corresponds with a more concave curve, with more of the mass shifted to the right. For example, on the second curve (Gini $=0.42$ ), we have $80 \%$ of the population controlling about $55 \%$ of the resources, whereas on the bottom curve (Gini $=0.77$ ), we have $80 \%$ of the population controlling only $20 \%$ of the resources.

In Economics, the Gini Index is frequently used to describe income disparities between countries. Reference [5], for example, lists Sweden at 0.23, Taiwan at 0.34, The UK at 0.40 , the US at 0.45 , Thailand at 0.53, and South Africa at 0.63.

Importantly, in this context, the Gini Index has no relationship with the net wealth of the country in question - a poor country may have less disparity than a rich one. For example, the Philippines and

\footnotetext{
6 We will use the terms Gini Coefficient and Gini Index interchangeably.

In some literature, the Gini Index is given in the range $[0,100]$. We are using the format more prevalent in the sciences,
} on the unit interval. the US have nearly identical Gini Indices for household income, despite a 10-fold difference in per-capita income, whereas per-capita income in Hong Kong, whose Gini Index is .537, is commensurate to that in the US [idem].

The Gini coefficient for the present subscriber population is about 0.42 ; interestingly, this is close to the income disparities seen in the USA and Western Europe [idem]. We also analyzed the subset of the population who had ever accessed the new web portal, revealing a nearly-identicallyshaped distribution (though with a larger mean), with a Gini coefficient of 0.38 .

In addition to unequal distribution of contacts (and thus, use of resources), we also noticed that the distribution is consistent with Pareto's Principle $^{7}$, which, in modern parlance, makes an assertion of both inequality and self-similarity within populations. Pareto's Principle states, roughly, that $80 \%$ of the wealth is controlled by $20 \%$ of the population (or is otherwise distributed unevenly and self-similarly). [14] Interestingly, the same statement applies to any contiguous subset of the population: for example, if we consider only the richest $20 \%$ of the population, we would expect wealth to be distributed in similar proportions (with $4 \%$ of the overall population controlling $64 \%$ of the wealth). In the case of the subscriber population, referring back to Fig. 5, we see that $50 \%$ of the population owns about $79 \%$ of the contacts. Nearly the same ratio is seen looking at the top $25 \%$ of the population or the bottom $20 \%$, or even some range in the middle [idem].

\section{BUILDING A NEW MODEL}

It was our desire to obtain a new statistical/analytical model for the present subscriber base. We analyzed a number of both well-known and esoteric distributions having central and tail behavior reasonably-similar to our data. Of these, we found the Log-Normal distribution to be almost the best fit. One or two exotic 3- or 4-parameter generalized power law functions had slightly better fits (by fractions of a percent); however, these distributions were notwellstudied or well-accepted in other population-study sciences, had no intuitive justification, and did not have tidy closed-form maximum likelihood estimators for their parameters.

8 Also referred to as Pareto Principle, Pareto's Law, $80 / 20$ law or rule, etc., and is sometimes what is being referred to when people discuss "The Long Tail". 
The Log-Normal distribution is related to the familiar Normal (or Gaussian) distribution. It appears frequently in the Social and Life Sciences and was suggested by [2] to model file sizes. As noted by [7], scientists often mistake LogNormally-distributed phenomena for Normal distributions having certain "outliers". Indeed, with a sufficiently small $\mathrm{N}$, the chances of seeing enough of the right tail to pique one's interest may be low. In our case, the tail mass and its consequences were abundantly-clear.

The Log-Normal probability distribution is defined as follows:

If random variable $X$ is Log-Normallydistributed, then random variable $Y=\ln (X)$ is Normally-distributed; equivalently, if $Y$ is Normally-distributed, then $e^{Y}$ is Log-Normallydistributed (where $\ln ()$ is the natural logarithm function and $e$ is the base of the natural logarithm).

The Log-Normal distribution is parameterized by (log-)scale $\mu € \Re$ and shape $\sigma>0$ and is supported over the positive reals. It has the curious property that its median is equal to its geometric mean, given by $\mathrm{e}^{\mu}$. Note that the median (110) and geometric mean (104) of our sample are quite close. And, unlike the Normal distribution, its mean is a function of both $\mu$ and $\sigma: \bar{x}=e^{\mu+\sigma^{2} / 2}$. [7], [8], [10].

Note also that the Log-Normal's shape parameter $\sigma$ is analogous to the standard deviation of the Normal Distribution, but is multiplicative versus additive. For example, given a Normal distribution with mean $\mu$ and standard deviation $\sigma$, the first confidence interval would be the range $[\mu-\sigma, \mu+\sigma]$ (covering $68.3 \%$ of the population); for a LogNormal distribution with median $\mathrm{e}^{\mu}$ and shape $\sigma$, the first confidence interval would be the range $[\mu / \sigma$, $\left.\mu^{*} \sigma\right]$ (also covering $68.3 \%$ of the population). The $i^{\text {th }}$ interval is given by $\left[\mu / \sigma^{\mathrm{i}}, \mu^{*} \sigma^{\mathrm{i}}\right]$ [7].

Computing the maximum likelihood estimates [9] (for a population) for $\mu$ and $\sigma$, is mercifully straight-forward with:

$$
\begin{aligned}
& \hat{\mu}=\frac{1}{n} \sum_{x \in X} \ln (x) \\
& \hat{\sigma}^{2}=\frac{1}{n} \sum_{x \in X}(\ln (x)-\hat{\mu})^{2}
\end{aligned}
$$

The values of these parameters for our population are shown in Table 1. These computations were simple enough that we were able to perform them within reasonably straightforward SELECT statements in Oracle $11 \mathrm{~g}^{\mathrm{TM}}$.

We now have a population model where a user's address book size is drawn from LogNormal ( $\hat{\mu}=4.6521, \hat{\sigma}=0.8427)$. Generating values from this distribution [10] requires only the ability to generate random values drawn from $\mathrm{Z} \sim$ Normal $(0,1)$ and then compute:

$$
\operatorname{LogNormal}(\mu, \sigma)=e^{\mu+\sigma Z}
$$

We find it useful to provide an intuitive justification for the LogNormal model. Recall from the Central Limit Theorem (CLT) that the mean of a set of independent, identically-distributed (i.i.d.) random variables tends towards a Normal distribution [idem]. An extension of the CLT into $\log$-space leads to a corollary that the product of a set of i.i.d. random variables tends towards the LogNormal distribution (recall that $\ln (\mathrm{a})+\ln (\mathrm{b})=$ $\ln (a b))$.

Returning to our subscriber model, suppose that the underlying distribution of address book sizes B is given by the product of a set of random variables relating to how address book sizes vary with the subscriber's age (A), income (I), and social connectedness (S), and some typical address book size $(\mathrm{Q})$ (which might be a constant). Then we have $\mathrm{B}=\mathrm{A} * \mathrm{I}^{*} \mathrm{~S} * \mathrm{Q}$. For example, a high school student may be more likely to participate in social networking, raising his expected address book size by a factor of 2.5; or, a wealthier person might be more likely to have a smart phone (capable of storing many contacts), contributing a factor of 5.4; or a less-socially-connected person might be expected to have fewer contacts, contributing a factor of 0.76 . We can imagine many other factors that might figure into this equation; the point, however, is that their effect is not additive, but multiplicative.

\section{MODEL VALIDATION}

To verify, at least in part, that our model accurately reflected production, we performed several goodness-of-fit tests on our derived analytic distribution versus our empirical data, including those shown in Fig. 6 and Fig. 7.

Comparing the CDFs of the empirical and predicted distributions directly and via a P-P plot (wherein the the CDFs are plotted against each other) [12], we see a very close match between our model and the population. The model is slightly 
heavier in its tails, but the overall correlation between the two data series (both coefficient of correlation and Pearson's product-moment correlation coefficient) was in excess of 0.999 . Other analyses of error, such as least-squares fit between the data series, showed that the model was within plus or minus $1.3 \%$ of the population.

Empirical and Predicted CDFs

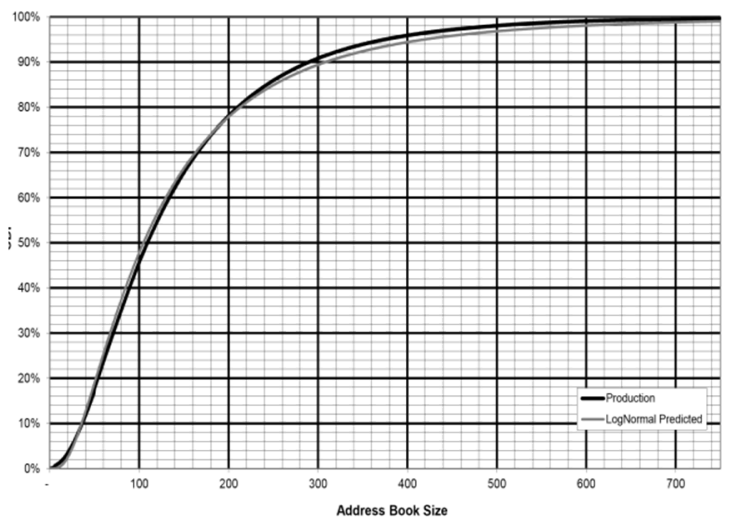

Fig. 6. Comparison of empirical subscriber base with that predicted by the LogNormal model.

Lognormal Model vs. Empirical Data P.P Plot

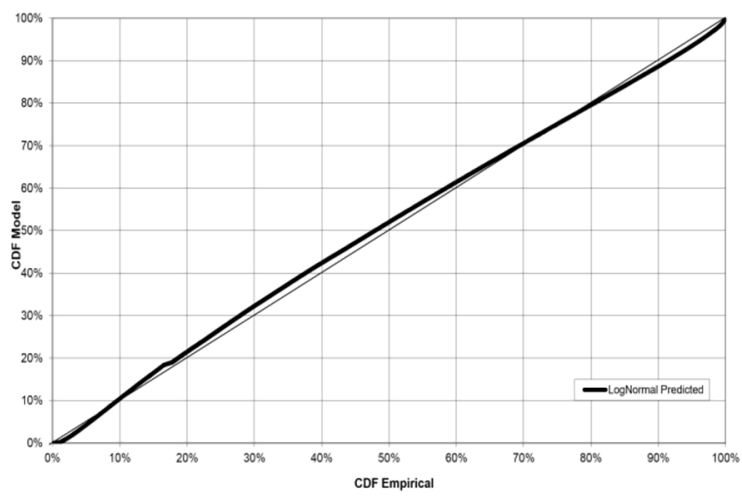

Fig. 7. P-P plot analyzing goodness-of-fit of the model against the population. If the fit were perfect, the $P-P$ plot would lie on the $y=x$ line.

\section{PARETO EVERYWHERE}

We found even greater disparity (higher Gini coefficients) in other areas of the population - akin to the difference in income inequality between the United States and South Africa (0.45 vs. 0.631) [5]. At the risk of over-extending the socio-economic metaphor, we might say that those in the wealthier upper-tail are also greater polluters. For example, change logs (analogous to transaction logs), which are generated each time a user makes a change to his address book, are stored on a file system; we found that about $10 \%$ of the population accounted for almost $60 \%$ of the change logs stored in the system. (The Gini coefficient for this distribution was 0.61.) A decision to enable a feature that, while expensive, concatenates and eliminates change logs (reducing the total number of files and total storage used), was made much easier: setting the threshold at which the feature is activated for a user such that only that top $10 \%$ of users addresses almost two thirds of the excess change logs in the system. This strategy proved successful.

We were also able to compute how frequently users made changes to their address books and compare this statistic with their address book sizes. We found that, across the board, a larger address book correlated with a higher change frequency (as estimated by the number of days since the last change). Again, the effect is multiplicative.

As alluded to earlier, we also examined the subset of the population who had used the new web portal; we found that the shape of the distribution of these users' address books was substantially the same as the larger population, but that they had, both at the median and on average, about 20 more contacts than non-web users.

We find, then, that users in the upper tail (with large address books) also perform more changes, perform transactions more frequently, and are more likely to use the web portal - thereby exposing them to latency induced by their data sizes. Each of these factors multiplicatively-magnifies the users' costs. It was surprising to us that the required capacity and scale of the system was driven so strongly by such a small percentage of its users. The design and/or product changes that this might motivate are beyond the scope of this article.

\section{CONCLUSIONS AND CONSEQUENCES}

Software systems offering demand-based resources to human users should expect to see heavy-tailed disparity in resource consumption among users. Models for simulation, testing, and monitoring such systems should therefore be designed accordingly.

The implementation of the systems themselves should be sensitive to the potential unboundedness of user requests. For example, the getAllContacts method discussed above, by default (and against our advice), literally returned all of the users contacts (and details thereof) to the caller; for the poor soul with 32,000 contacts (thanks to 
integration with social media), this meant a long wait for his portal to load. This method was changed to require paging of results (e.g., fetching $\mathrm{N} \leq 50$ contacts at a time) which, in turn, provided (more) deterministic latency bounds and user experience. The value of data caching, parallelization, and other optimization techniques should be considered carefully in light of the application and its usage model.

The first-order statistics that we have all come to know and love - min, max, mean, standard deviation, etc., are misleading and/or useless in the presence of certain human-behavioral phenomena. At minimum, the median should always be examined in comparison to the mean; when they frequently differ by a significant amount, rank-andorder statistics (e.g., percentiles) will provide the full picture. This may require building or enhancing monitoring and analysis tools, but it is unavoidable if an accurate understanding of user demand is desirable.

In like manner, it is unwise to make statements about average or worst-case performance in such systems: averages are pessimistically-misleading and maxima can be essentially unbounded. Instead, nameplate ratings, KPIs, SLAs, and the like are more-appropriately specified by performance at specific percentiles (e.g., median response time shall be less than $100 \mathrm{~ms}$ and $95^{\text {th }}$-percentile response time shall not exceed $500 \mathrm{~ms}$ ).

Users on the long right tail will often cause bursty traffic; in the present case, this manifested as high $\mathrm{CPU}$ and I/O utilization at the various components servicing the user's transaction. Such behavior is only visible if system metrics (such as CPU utilization) are collected at a relatively high frequency. At lower frequency, one is effectively taking averages over the longer intervals, thus hiding bursty (long-tail) behavior. Minimizing this averaging effect makes rank-and-order analysis more furtive.

Finally, there are humans using the system; the more-demanding users are more likely to be sensitive to performance issues and more likely to cause performance issues, for example, by using more computationally- or storage-intensive features. This case-study provides several examples of how power-users can cause subtle problems to become magnified - potentially to the extent of impacting other users' experiences.

Said more briefly, disparity leads to more disparity; expect to come head-to-head with Pareto's Principle and address it appropriately.

\section{REFERENCES}

[1] Andrew S. Tanenbaum, Jorrit N. Herder, Herbert Bos. "File size distribution on UNIX systems - then and now" (2006). See also: http://www.minix3.org/docs/jorrit-herder/osrjan06.pdf.

[2] Downey, A. "The structural cause of file size distributions". IEEE MASCOTS 2001.

[3] Crovella, M.E.; Bestavros, A., "Self-similarity in World Wide Web traffic: evidence and possible causes," Networking, IEEE/ACM Transactions on, vol.5, no.6, pp.835,846, Dec 1997. doi: $10.1109 / 90.650143$

[4] Mell, Peter, and Timothy Grance. "The NIST definition of cloud computing." (2011).

[5] Factbook, C. I. A. "The world factbook." See also: https://www. cia.

gov/library/publications/the-world-factbook (2010).

[6] Gastwirth, Joseph L. "The estimation of the Lorenz curve and Gini index." The Review of Economics and Statistics 54, no. 3 (1972): 306316. See also:

http://www.jstor.org/stable/pdfplus/1937992.p df.

[7] Limpert, Eckhard, Werner A. Stahel, and Markus Abbt. "Log-normal distributions across the sciences: keys and clues." BioScience 51, no. 5 (2001): 341-352.

[8] Crow, Edwin L., and Kunio Shimizu. Lognormal distributions: Theory and applications. Vol. 88. CRC PressI Llc, 1988.

[9] Cohen, A. Clifford, and Betty Jones Whitten. "Estimation in the three-parameter lognormal distribution." Journal of the American Statistical Association 75, no. 370 (1980): 399404.

[10] Aitchison, J. and Brown, J.A.C. The Lognormal Distribution, Cambridge University Press (1957).

[11] "Apache HTTP server version 2.2 documentation". Apache Software Foundation (2013). Accessed 19 April 2013. See also: http://httpd.apache.org/docs/2.2/logs.html\#acce sslog

[12] Wilk, Martin B., and Ram Gnanadesikan. "Probability plotting methods for the analysis for the analysis of data." Biometrika 55, no. 1 (1968): 1-17.

[13] Kim, Bong-Ho, Jungnam Yun, Yerang Hur, Chakchai So-In, Raj Jain, and A-K. Al Tamimi. "Capacity estimation and TCP performance enhancement over mobile WiMAX networks." Communications Magazine, IEEE 47, no. 6 (2009): 132-141. 
A Blate and K Jeffay / International Journal of Computer Networks and Communications Security, 1 (1), JUNE 2013

[14]Newman, Mark EJ. "Power laws, Pareto distributions and Zipf's law."Contemporary physics 46, no. 5 (2005): 323-351. 